This item was submitted to Loughborough's Research Repository by the author.

Items in Figshare are protected by copyright, with all rights reserved, unless otherwise indicated.

\title{
Privacy-preserving dialogues between agents: a contract-based incentive mechanism for distributed meeting scheduling
}

\section{PLEASE CITE THE PUBLISHED VERSION}

https://doi.org/10.1007/978-3-030-66412-1_19

\section{PUBLISHER}

Springer

VERSION

AM (Accepted Manuscript)

\section{PUBLISHER STATEMENT}

The final authenticated version is available online at https://doi.org/10.1007/978-3-030-66412-1_19.

\section{LICENCE}

CC BY-NC-ND 4.0

\section{REPOSITORY RECORD}

Di, Boya, and Nick Jennings. 2021. "Privacy-preserving Dialogues Between Agents: A Contract-based Incentive Mechanism for Distributed Meeting Scheduling". Loughborough University.

https://hdl.handle.net/2134/16930663.v1. 


\title{
Privacy-Preserving Dialogues between Agents: A Contract-Based Incentive Mechanism for Distributed Meeting Scheduling
}

\author{
Boya Di and Nicholas R. Jennings \\ Department of Computing, Imperial College London, London, UK \\ \{b.di, n.jennings\}@imeprial.ac.uk
}

\begin{abstract}
Meeting scheduling (MS) is a practical task in everyday life that involves independent agents with different calendars and preferences. In this paper, we consider the distributed MS problem where the host exchanges private information with each attendee separately. Since each agent aims to protect its own privacy and attend the meeting at a time slot that it prefers, it is necessary to design a distributed scheduling mechanism where the privacy leakage can be minimized and as many agents are satisfied with the outcome as possible. To achieve this, we propose an intelligent two-layer mechanism based on contract theory where the host motivates each agent to reveal its true preferences by providing different rewards without knowing the costs of each agent to attend the meeting. We first model the privacy leakage by measuring the difference between the revealed information of an agent's calendar and other agents' prior beliefs. An optimal control problem is then formulated such that the reward function and privacy leakage level can be jointly designed for each agent. Through theoretical analysis, we show that our proposed mechanism guarantees the incentive compatibility with respect to all agents. Compared to the state of the art, empirical evaluations show that our proposed mechanism achieves lower privacy leakage and higher social welfare within a small number of rounds.
\end{abstract}

Keywords: Meeting scheduling - Incentive mechanism · Privacy.

\section{Introduction}

Many multi-agent systems involve reaching an agreement between self-interested agents that seek to minimize the amount of information revealed to their opponents. Examples include electronic commerce, computer games and meeting scheduling. In this paper, we focus on multi-agent meeting scheduling (MAMS) as a representative application. In this case, the host of a meeting is required to arrange a staring time that is acceptable to all attendees. In practice, the MAMS problem is inherently a distributed one since the agents' calendars are different and private, meaning that they are unwilling to share them with others. Given this situation, there is a clear trade-off between maximizing social welfare and minimizing the amount of information revealed in this process [1]. 
Against this background, we study the distributed MAMS problem in a setting where the host proposes candidate time slots in each round and attendees respond to the proposals separately. Since all agents are self-interested, each of them desires the meeting to be scheduled at one of its preferable time slots while revealing as little private information as possible. In such cases, it is necessary to design an incentive mechanism with the following desirable features. First, the mechanism should be incentive compatible to avoid the case where a selfish agent can manipulate the scheduled meeting for its personal benefit. Second, privacy leakage should be minimized and the mechanism should have a fast convergence speed. Third, the social welfare should be maximized, meaning, as many agents as possible should be satisfied with the scheduled time slot.

A useful tool to handle the trade-off between incentive compatibility and privacy preservation is economic contract theory [2], in which agreements are designed to motivate agents with conflicting interests to accept mutually beneficial offers. This mathematical tool provides an efficient approach to incentivization by offering contracts to each type of agent (classified by the agents' private attributes). Here a contract consists of a required action and a corresponding reward. Given properly designed rewards, the agent only needs to choose the contract aligned with its own type to maximize its utility.

In this paper, we propose a distributed two-layer contract-based incentive mechanism where the host offers different rewards in the forms of tokens or credits to motivate attendees to reveal true preferences ${ }^{1}$. Such forms of rewards are widely used in on-line membership [3], resource trading [4], and blockchain systems [5]. Each attendee first selects one outer-layer contract from a candidate set provided by the host, which defines 1) a tailored set of rewards paid to each attendee corresponding to different preferences, and 2) the number of proposals each attendee is required to respond to at each round (i.e., the privacy leakage level). For each proposed time slot, the host provides attendees with multiple agent-specific inner-layer contracts, each of which consists of a reward along with the required action (e.g., attend/not attend the meeting, hold the offer, etc.).

As such, we advance the state-of-the-art in the following ways.

- We develop a novel metric to define the privacy leakage in a general way such that it can depict both privacy leakage and the amount of protected private information. By leveraging the probability distribution of an agent's availability, we measure privacy as the difference between other agents' prior belief and the agent's desired belief that it wishes to reveal to others.

- We are the first to develop a privacy-preserving incentive mechanism in the context of MAMS where the privacy leakage level can be optimized based on each agent's calendar.

- We achieve a minimum incentive cost by properly designing the rewards and allowing the agents to have multiple types of responses. This is the first attempt to jointly optimize the reward functions and privacy leakage levels via an optimal control method.

\footnotetext{
${ }^{1}$ An agent's availability at each time slot reflects its preference over the time slots, which can be categorised as multiple types, such as 'free', 'OK with it', and 'busy'.
} 
- Simulation results show that the proposed mechanism saves $58.3 \%$ privacy leakage compared to the calendar-sharing scheme. Compared to current state-of-the-art negotiation mechanism, it also achieves a better trade-off between the privacy leakage and the convergence speed. To achieve the same convergence speed, the contract mechanism can reduce $26.2 \%$ privacy leakage; for a same level of privacy leakage, the contract mechanism saves $80 \%$ time costs for convergence. It achieves over $88.31 \%$ of the optimal social welfare obtained by a centralized method, and significantly outperforms existing works by up to 82 percentage points. By allowing multiple types of responses, the mechanism reduces the incentive cost by up to $18 \%$.

Existing literature on distributed MAMS problems explore various schemes such as negotiation-based methods, Max-Sum algorithms and its variants for distributed constraint optimization problems (DCOP), and incentive mechanisms for consensus. For instance, the works of [6] and [7] consider automated negotiation between the host and attendees. Agents' preferences were quantified and different negotiation strategies were developed to improve the social welfare. By formulating the MAMS problem as a DCOP, the Max-Sum algorithms proposed in [8] and local consistency reinforcement [9] can be utilized where the solution space is traversed indirectly via limited private information exchange among all agents. In the work of [10], the extended VCG mechanism finds the optimal strategy of each agent, which is aligned with the solution of social welfare maximization. Agents share all their calendar information with each other. However, these works have not linked privacy and incentive compatibility. The developed methods either rely on trust between agents [6-9] or agents' willingness to share their private information with others $[8,10]$. Neither of these extremes is suitable to solve our problem.

The mechanism we propose is also related to the literature on contract theory ${ }^{2}$ and game theory where most works [11-13] focus on incentives in MAS with information asymmetry. For instance, the work of [11] concerns the task assignment problem where multiple contracts are offered to agents such that each agent only picks one that it is capable of to achieve the maximum utility. The work of [13] studies the privacy issue in multi-agent data collection. Privacy is considered as a type of service and assigned to agents for utility maximization. Nevertheless, this line of works assumes that the host is trustworthy and only cares about the incentive compatibility with respect to attendees.

The rest of the paper is organized as below. In Section 2 we formulate the distributed MAMS problem where privacy leakage is modelled. In Section 3 we design a contract-based MAMS protocol to reach a consensus. The reward functions and privacy leakage level for each agent are jointly optimized via solving an optimal control problem in Section 4. Theoretical analysis on the proposed mechanism is also provided. In Section 5, we evaluate our proposed mechanism by simulations. Finally we conclude in Section 6 .

\footnotetext{
${ }^{2}$ It is worth noting that contract theory is different from the contract network
} protocol. The latter is a type of negotiation-based mechanism for task assignment. 


\section{Meeting Scheduling Problem Formulation}

\subsection{Problem Definition}

The distributed MSP consists of multiple agents and a number of meetings to be scheduled. We consider a dynamic case where the need for new meetings arises randomly and is not known by the agents in advance. Given a set of meetings and a group of $N$ agents $\mathcal{A}$, we define each meeting $m$ by a tuple

$$
m=\left\{\mathcal{A}_{m}, A_{0}, \mathcal{T}, l, t\right\},
$$

where $\mathcal{A}_{m}$ is the subset of agents to attend meeting $m, A_{0}$ is the agent who will host the meeting and propose candidate time slots to other agents, $\mathcal{T}$ is the set of time slots during which the meeting is expected to be held, and $l$ is the length of meeting $m$ (i.e., the number of time slots), $t$ is the starting time of the meeting. In other words, the starting time $t$ will be selected from $\mathcal{T}$ and $l$ slots will be reserved for meeting $m$ if scheduled.

A meeting is scheduled via the propose-and-respond process where in each round the host proposes multiple time slots to each attendee and receives responses. We classify the responses of each agent as three states ${ }^{3}$ based on its availability: "I am free", "I am OK with this slot", or "I am busy". By replying OK, the attendee holds the offer temporally and expects the host to propose other time slots. It only accepts slot $t$ if no other feasible solution to the MAMS problem is found. We denote the set of three possible states as $\mathcal{S}=[F, O, B]$. The real state and reported state of each attendee $i$ with respect to time slot $t$ are denoted as $r_{i}(t) \in \mathcal{S}$ and $s_{i}(t) \in \mathcal{S}$. We refer to one time slot as a free/OK/busy slot if the agent's real state is free/OK/busy at this slot.

If the meeting is scheduled at slot $t$, each attendee $i$ 's utility is given below

$$
U_{i}\left(t, s_{i}(t), R_{r_{i}(t)}\right)=R_{r_{i}(t)}-C_{i}^{s_{i}(t), r_{i}(t)}-\alpha L_{i}\left(r_{i}(t)\right),
$$

- $R_{r_{i}(t)}$ is the reward (in the forms of tokens or credits) paid by the host when the reported state of attendee $i$ is $r_{i}(t)$;

$-C_{i}^{s_{i}(t), r_{i}(t)}$ is the cost of attendee $i$ to report its state as $r_{i}(t)$ given its actual state $s_{i}(t)$. For example, $C_{i}^{O, F}$ is the cost of agent $i$ to attend the meeting when it is OK with slot $t$.

- $L_{i}\left(r_{i}(t)\right)$ is the privacy leakage of attendee $i$, and $\alpha$ depicts its sensitivity towards privacy.

Similarly, the host's utility can be given by

$$
U_{0}\left(t,\left(R_{r_{i}(t)}\right)_{i \in \mathcal{A}}\right)=\left\{\begin{array}{l}
\Gamma-\sum_{1 \leq i \leq N} R_{r_{i}(t)}-C_{0}^{s_{0}(t), F}-\alpha L_{0}\left(r_{0}(t)\right), \forall r_{i}(t) \neq B \\
-\infty, \exists r_{i}(t)=B
\end{array}\right.
$$

where $\Gamma$ measures the satisfaction of the host for successfully scheduling a meeting. The above equation omits parameter $m$ for convenience.

\footnotetext{
3 This can be extended to more types, but we use three to keep the example simple.
} 


\subsection{Agents' Preferences and Privacy Leakage}

Agents' Preferences. Each agent $i$ 's preference over different time slots is related to its state. For example, agent $i$ prefers slot $t$ to $t^{\prime}$ if it is free at slot $t$ and OK with slot $t^{\prime}$. The cost function $C_{i}^{s_{i}(t), r_{i}(t)}$ is utilized to quantify each agent's preferences, which influences agent $i$ 's response strategy. The ordering of costs for each agent $i$ is determined by the pair of states $\left(s_{i}(t), r_{i}(t)\right)$ :

$$
C_{i}^{B, F}>C_{i}^{B, O} \gg C_{i}^{O, F} \gg C_{i}^{F, F}>C_{i}^{O, O}=C_{i}^{F, O}>C_{i}^{B, B}=C_{i}^{F, B}=C_{i}^{O, B}=0 .
$$

$-C_{i}^{s, r}=0, r=B$ : When the agent reports it is busy at one slot, it does not need to attend the meeting. Thus, the cost is 0 regardless of its real state.

$-C_{i}^{O, O}=C_{i}^{F, O}$ : The agent's cost of reporting OK is the same no matter whether it is actually free or OK at this time slot, since it is not necessary for the agent to attend the meeting in both cases.

$-C_{i}^{O, O}<C_{i}^{F, F}$ : An agent's cost to tell the truth about an OK slot is lower than its cost of truth-telling with respect to a free slot, since it is required to guarantee the attendance at the OK slot.

$-C_{i}^{B, F}>C_{i}^{B, O} \gg C_{i}^{O, F} \gg C_{i}^{F, F}$ : For agent $i$, its cost of attending the meeting at a busy slot is higher than that of claiming to be OK with this slot, which is also much higher than its cost at a free or OK slot regardless of its response. In other words, the fact that an agent cannot attend the meeting at a busy slot is represented by a high cost. Similarly, its cost of attending the meeting at an OK slot is higher than that at a free slot.

We assume that the cost of each agent $i$ depends on how much availability it has throughout the calendar, i.e., the density of availability, which can be depicted by $d_{i}$. In other words, the busier an agent is, the higher its cost to attend a meeting. We define $d_{i}$ by the number of free and OK time slots,

$$
d_{i}=\left(\frac{N_{F, i}}{N}\right)^{\beta_{1}}+\left(\frac{N_{O, i}}{N}\right)^{\beta_{2}},
$$

where $N_{F, i}$ and $N_{O, i}$ are the number of free and OK time slots, $N$ is the total number of candidate slots, and $\beta_{1}$ and $\beta_{2}$ are scaling factors. We set $\beta_{2}>\beta_{1}>0$ and $\beta_{1}, \beta_{2} \notin \mathbb{Z}$ such that each pair of $\left(N_{F, i}, N_{O, i}\right)$ is mapped to one unique density value $d_{i}$, which is considered as agent $i$ 's type. A general form of the cost function is then given by

$$
C_{i}^{s, r}\left(d_{i}\right)=\frac{a_{1}^{s, r}}{\left(1+d_{i}\right)^{a_{2}^{s, r}}}+a_{3}^{s, r},
$$

where $a_{k}^{s, r}>0$ is a parameter $(k=1,2,3)$. Following rule (4), the family of cost functions for each agent $i$ can then be generated via (6).

Privacy Leakage. We consider the case where each attendee only communicates with the host and does not know any detail of other attendees. Therefore, 
the privacy information that each attendee (or the host) leaks is its calendar information revealed to the host (or the attendee).

Before the propose-and-respond process, the host and each attendee has a prior belief about the probability that the other is free at each time slot. As the process progresses, each attendee (or the host) gets new information from the host (or each attendee) and updates probability information based on the proposal (or the response). Note that each agent has different attitudes towards the privacy information. For those who tend to protect their calendar, they expect the host to believe that their probability of being free is 0.5 (i.e., not sure about its availability). In contrast, other agents might prefer others to have an impression that they are busy (or available) even if they are not.

To depict such diversity of agents' attitudes, we utilize the difference between one agent's expected probability distribution and other's belief, namely, statistical distance, to measure the privacy leakage. We denote the desired probability of being free at time slot $t$ that agent $j$ wishes others to believe and the prior belief that agent $i$ has about agent $j$ 's availability at this slot as $p_{i \rightarrow j}^{d}(t)$ and $p_{i \rightarrow j}^{b}(t)$, respectively. After this time slot $t$ is proposed, the updated probability is denoted by $p_{i \rightarrow j}^{a}\left(t, r_{i}(t)\right)$. The privacy leakage $L_{i}\left(r_{i}(t)\right)$ is given by

$$
L_{i}\left(r_{i}(t)\right)=\left|p_{i \rightarrow j}^{b}(t)-p_{i \rightarrow j}^{d}(t)\right|-\left|p_{i \rightarrow j}^{a}\left(t, r_{i}(t)\right)-p_{i \rightarrow j}^{d}(t)\right| .
$$

For $L_{i}\left(r_{i}(t)\right)>0$ and $L_{i}\left(r_{i}(t)\right)<0$, it measures leaked privacy and protected privacy, respectively.

Remark 1. The proposed privacy leakage metric can readily depict the agents' different attitudes towards their privacy information:

$-p_{i \rightarrow j}^{d}(t)=0.5$ : agent $i$ is privacy-negative, i.e., it does not want others to know anything about its calendar;

- $p_{i \rightarrow j}^{d}(t)=1$ (or $p_{i \rightarrow j}^{d}(t)=0$ ): agent $i$ is privacy-active, implying that it desires to leave others the impression that it has a clear/busy calendar;

$-p_{i \rightarrow j}^{d}(t)$ is set as the real probability of agent $i$ : the agent is privacy-neutral, i.e., it does not care about how others view its calendar.

\section{Contract-Based Meeting Scheduling Protocol}

To motivate each attendee to reveal their availability at the proposed time slot, the host provides different rewards depending on the attendee's response. When a meeting is set at time slot $t$, an attendee is offered a high reward if it reports to be free and guarantees to attend the meeting. In contrast, it gets a medium reward if it reports to be OK and requires the host to spend more resources to explore other slots before finally accepting slot $t$. Given each attendee's cost functions, the rewards can be designed in a way that each attendee can only get the highest utility when it tells the truth.

However, the cost functions are unknown to the host, which is determined by each agent's type, i.e., the density of availability as shown in (2). Examples 


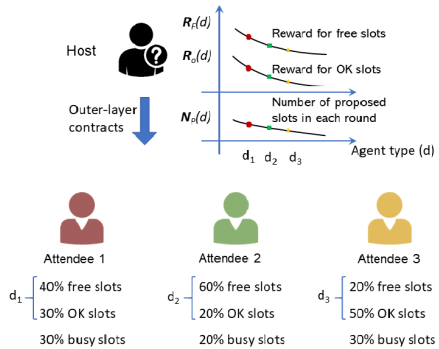

(a) Outer-layer contracts

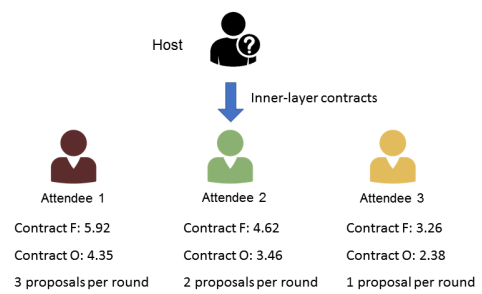

(b) Inner-layer contracts

Fig. 1. Illustration of the contract-based mechanism

of different types of agents are given in Fig. 1(a). The busier an attendee is (i.e., the value of $d$ is smaller in Fig. 1(a)), the higher its cost to attend the meeting, thereby requiring a higher reward. To avoid the attendee claiming to be busy to get high rewards, the host requires those busy attendees to respond to a larger number of proposed time slots in each round, leading to a higher privacy leakage level. Since each attendee is privacy-preserving ${ }^{4}$, they are not willing to lie at the cost of higher privacy leakage. Following this insight, the reward function for each agent type, as well as the number of proposed slots, can be properly designed to keep them incentive compatible. For example, three red (or green) points on the three curves in Fig. 1(a) shows rewards and the number of proposed slots for an type- $d_{1}$ (or $d_{2}$ ) agent.

Based on the above intuitions, the detailed protocol is shown below.

1. When the need to schedule meeting $m$ arrives, the host publishes the meeting information to the group of attendees.

2. Each attendee labels the valid starting time slots 'free', 'OK', and 'busy', and keeps it as private information along with its cost at each slot.

3. The host sends a menu of outer-layer contracts to each attendee, denoted by $\left\{\left(R_{F}(d), R_{O}(d), N_{p}(d)\right)\right\}$, where $d$ represents the type of an agent, identified by its density of availability as shown in (5). The menu is shown in Fig. 1(a). The number of contracts contained in the menu equals the number of agents' types. Each contract requires the agent to respond to on average $N_{p}(d)$ proposed time slots at each round, and will receive a reward $R_{F}(d)$ or $R_{O}(d)$ if the meeting is scheduled at a free or OK slot.

4. Each attendee $i$ selects one outer-layer contract from the menu to maximize its expected utility received from the coming propose-and-respond process. For example, in Fig. 1(a) attendee 1 may select the outer-layer contract represented by three red circles.

5. According to the signed out-layer contract, the propose-and-respond process starts. In each round as shown in Fig. 1(b), the host proposes $N_{p}\left(d_{i}\right)$ time

\footnotetext{
${ }^{4}$ Here we assume that every agent cares about its privacy to the same degree, which
} also makes sense in reality. 
slots to each attendee. For each time slot $t$, the host offers three innerlayer contracts. 1) Contract $F$ offers a high reward $R_{F}\left(d_{i}\right)$ and requires the attendee to attend the meeting. 2) Contract $O$ offers a medium reward $R_{O}\left(d_{i}\right)$ and allows the attendee to attend the meeting at slot $t$ only when there is no other options. 3) Contract $B$ offers zero reward to the attendee who is busy at slot $t$. In Fig. 1(b), we omit the zero-reward contract $B$ for simplicity.

6. Each attendee selects one inner-layer contract to maximize its utility shown in (2) and responds to the host. If an attendee reports to be OK with one slot, the host is required to explore at least one more slot in the next round.

7. Steps 5 and 6 are repeated until either one time slot is found in which at least $N_{t}$ attendees are free or the maximum number of rounds $M$ is reached. After $M$ rounds, the host selects one slot $t$ where the largest number of attendees is free, denoted by $N_{f}(t)$. The host then raises the rewards for $N_{t}-N_{f}(t)$ OK attendees so as to maintain its reputation.

The reasoning behind Step 7 will be illustrated in Section 4.2.

\section{Two-layer Contract Design}

In this section, we first optimize the reward functions $\left(R_{F}(d), R_{O}(d)\right)$ and privacy leakage level $N_{p}(d)$. Properties of the mechanisms are then analysed.

\subsection{Joint Reward and Privacy Leakage Optimal Control}

\section{Attendee's strategy.}

When each responder $n$ selects the outer-layer contract, it does not know in which time slot the meeting will be scheduled yet. Therefore, it makes the decision by maximizing its expected utility. Attendee $i$ 's expected utility when a free/OK/busy slot is finalized for the meeting is given below.

$$
\begin{aligned}
E\left[U_{e}\left(\hat{d}, s_{i}\right)\right] & =f_{s_{i}}\left(d_{i}\right) \cdot \max _{r_{i} \in \mathcal{S}}\left[R_{r_{i}}(\hat{d})-C^{s_{i}, r_{i}}\left(d_{i}\right)-\alpha N_{p}(\hat{d}) L_{i}\left(r_{i}\right)\right] \\
& =f_{s_{i}}\left(d_{i}\right) \cdot U_{e}\left(\hat{d}, s_{i}\right)
\end{aligned}
$$

where $f_{s_{i}}\left(d_{i}\right)=N_{s_{i}, i} / N_{i}$ maps $d_{i}$ to the density of free (or OK or busy) time slots as shown in equation (5), and $\alpha N_{p}(\hat{d}) L_{i}\left(r_{i}\right)$ is the minimum accumulated privacy leakage. Note that $\alpha$ decreases with the increase of each attendee's calendar density, which can be depicted by a quadratic function. For convenience, we omit the subscript $t$ here. Therefore, the expected utility of attendee $i$ can be given by

$$
E\left(\hat{d} ; d_{i}\right)=\sum_{s_{i} \in \mathcal{S}} E\left[U_{e}\left(\hat{d}, s_{i}\right)\right]
$$

and each attendee's strategy is to pick a type- $\hat{d}$ outer-layer contract to maximize the above expected utility. 
Incentive compatible constraints of attendees. To ensure that each attendee $i$ selects the $d_{i}$-type outer-layer contract designed for it rather than other types, the following incentive compatible constraint should hold

$$
\max _{\hat{d}} E\left(\hat{d} ; d_{i}\right)=E\left(d_{i} ; d_{i}\right), \forall i .
$$

The sufficient conditions to satisfy this constraint can be given by

$$
\begin{aligned}
& R_{F}(\hat{d})-R_{M}(\hat{d})>C^{F, F}\left(d_{i}\right)-C^{O, O}\left(d_{i}\right)+\alpha P_{O} \cdot N_{p}(\hat{d}), \\
& R_{F}(\hat{d})-R_{M}(\hat{d})<C^{O, F}\left(d_{i}\right)-C^{O, O}\left(d_{i}\right)+\alpha P_{O} \cdot N_{p}(\hat{d}), \\
& f_{F}\left(d_{i}\right) \cdot U_{e}(\hat{d}, F)+f_{O}\left(d_{i}\right) \cdot U_{e}(\hat{d}, O) \leq f_{F}\left(d_{i}\right) \cdot U_{e}\left(d_{i}, F\right)+f_{O}\left(d_{i}\right) \cdot U_{e}\left(d_{i}, O\right),
\end{aligned}
$$

where $P_{O}$ is the probability of an agent being free at an OK slot. The typical value is 0.5 . We ignore $U_{e}(\hat{d}, F)$ here since it is usually rather small $\left(R_{B}=C^{B, B}=0\right)$.

When the outer-layer contract is selected, the propose-and-respond process starts. To motivate each attendee to report their true availability, the following constraint should be satisfied:

$$
\max _{r_{i}(t) \in \mathcal{S}} U_{i}\left(t, s_{i}(t), R_{r_{i}(t)}\right)=U_{i}\left(t, s_{i}(t), R_{s_{i}(t)}\right) .
$$

We derive its equivalent conditions as

$$
\begin{aligned}
& R_{O}(d) \geq C^{O, O}(d)+\alpha P_{O}+\epsilon, \\
& R_{F}(d) \geq R_{O}(d)+\max _{d}\left[C^{F, F}(d)-C^{O, O}(d)\right]+\alpha+\epsilon .
\end{aligned}
$$

where $\epsilon>0$ is a number small enough, and $p_{O} \leq 0.5$ is the probability of an attendee to be free at an OK time slot.

Optimal control of the host. The host offers a set of outer-layer contracts to attendees without knowing their types. Its objective is to find an optimal menu of contracts which minimizes the incentive cost and its own privacy leakage subject to all constraints listed above. The problem can be formulated below:

$$
\begin{aligned}
& \min _{\mathbf{R}(\cdot), N_{p}(\cdot)} \int_{\underline{d}}^{\bar{d}}\left[f_{F}(d) R_{F}(d)+f_{O}(d) R_{O}(d)+\alpha N_{p}(d) L_{0}(F)\right] g(d) d d \\
& \quad \text { s.t. }(11),(13),
\end{aligned}
$$

where $g(d)=1 / N_{t h}$ is the probability density function of $d$, following a uniform distribution and $N_{t h}$ is the number of possible values of $d$. The upper and lower bounds of $d$ are given by $\bar{d}$ and $\underline{d}$.

Though described with one simple inequality, (11c) actually implies a huge family of constraints, each of which corresponds to a certain pair of $\hat{d}$ and $d_{i}$. To identify the set of feasible solutions to the above problem, we simplify this constraint by its relaxed version shown in Proposition 1. The proof can be found in the Appendix A. 
Proposition 1. Constraint (11c) can be relaxed by the following constraints:

$$
\begin{gathered}
\frac{d R_{F}(d)}{d d} \leq 0, \\
\frac{d R_{O}(d)}{d d} \leq 0 \\
\frac{1}{\alpha L(F)} \frac{d R_{F}(d)}{d d}=\frac{1}{\alpha L(O)} \frac{d R_{O}(d)}{d d}=\frac{d N_{p}(d)}{d d},
\end{gathered}
$$

The formulated problem is an optimal control problem to find an optimal function rather than a value. Based on the Pontryagins maximum principle [14] as well as constraints $(11 a),(11 b)$, and (13), the numerical forms of both reward and privacy leakage functions can be obtained.

\subsection{Properties of the Mechanism}

Privacy preservation. As shown in (8) and (14a), an optimal privacy leakage level exists for each agent to reach a balance between the amount of leaked information and its social welfare.

Incentive Compatibility. We investigate the behaviours of attendees and the host separately to show the incentive compatibility of the proposed mechanism.

Definition 1. In the MAMS setting, an incentive compatible mechanism motivates attendee $i$ to always reveals its true preference over the proposed time slots to maximize its utility, i.e.,

- When selecting the outer-layer contracts, attendee $i$ selects the type- $d_{i}$ contract to maximize its expected utility, as shown in (10).

- In the propose-and-response process, if the meeting is scheduled at slot $t$, attendee $i$ can only obtain the maximum utility when they tell the truth, i.e.,

$$
\arg \max _{r_{i}(t) \in \mathcal{S}} U_{i}\left(t, s_{i}(t), R_{r_{i}(t)}\right)=s_{i}(t) .
$$

- In the propose-and-response process, the attendee has no incentive to manipulate the meeting to be scheduled at time slot $t^{\prime}$ by lying about its availability at slot $t$, i.e.,

$$
E\left[U_{i}^{l i e}\left(t^{\prime}, s_{i}\left(t^{\prime}\right), R_{s_{i}\left(t^{\prime}\right)} \mid r_{i}(t) \neq s_{i}(t)\right)\right] \neq E\left[U_{i}^{t r u}\left(t, s_{i}(t), R_{s_{i}(t)}\right)\right] .
$$

Based on Definition 1, we present Proposition 2 as proved in Appendix B.

Proposition 2. The proposed mechanism is incentive compatible with respect to all attendees.

For the host, there might exist a conflict between its own preference and others' social welfare, leading to unfaithful behaviours. For example, when the host is free at the proposed time slot $t^{\prime}$ while all attendees report to be either free or OK, it may claim that it is busy at all other time slots so as to schedule the meeting at time slot $t^{\prime}$ by ignoring those OK attendees' will. 
Definition 2. An mechanism is incentive compatible with respect to the host if it cannot get higher expected utility by lying about its availability at time slot * $^{*}$ in meeting $m$, i.e.,

$$
\begin{aligned}
& U_{0}\left(m, t^{\prime}, R_{r_{i}\left(t^{\prime}\right)} \mid r_{0}\left(t^{*}\right) \neq s_{0}\left(t^{*}\right)\right)+E_{m^{\prime}}\left[U_{0}\left(m^{\prime}, t, R_{r_{i}(t)} \mid m, r_{0}\left(t^{*}\right) \neq s_{0}\left(t^{*}\right)\right)\right] \leq \\
& U_{0}\left(m, t, R_{r_{i}(t)} \mid r_{0}\left(t^{*}\right)=s_{0}\left(t^{*}\right)\right)+E_{m^{\prime}}\left[U_{0}\left(m^{\prime}, t, R_{r_{i}(t)} \mid m, r_{0}\left(t^{*}\right)=s_{0}\left(t^{*}\right)\right)\right]
\end{aligned}
$$

where $t^{\prime}$ is the time slot that the host prefers to hold the meeting $m$. This implies that the host's expected utility will reduce in future meetings $\left\{m^{\prime}\right\}$.

Observation 1. An attendee who reports OK at slot $t$ expects the host to propose other time slots. If the attendee does not trust the host to do so, it reports it is busy. This leads to a higher probability of scheduling failure, which brings negative utility to the host.

Observation 2. Once the attendees do not trust the host, the host needs to provide a new inner-layer contract $F^{\prime}$ with a higher reward $R_{F}^{\prime}$ below to motivate the OK attendee to attend the meeting.

$$
R_{F}^{\prime}(d) \geq C^{O, F}(d)+\alpha P_{O}-\alpha+\epsilon .
$$

This guarantees the meeting to be scheduled successfully at the host's preferred time slot at an expense of a higher incentive cost.

The above observations imply that once an OK attendee finds the host not trust-worthy, it lies about its availability and has less chance to be scheduled at a free slot, i.e., its social welfare is degraded. The host then needs to offer higher rewards to successfully schedule the meeting.

Remark 2. The interaction between the host and an attendee OK with time slot $t$ in the propose-and-respond process can be formulated as a non-cooperative repeated game. Only when they both tell the truth, they each gets the maximum utility. Since we have a dynamic setting that new meetings arrive randomly and are not known to agents, this is an infinite repeated game.

Proposition 3. In an infinite repeated game of MAMS, the optimal strategy for both the host and attendees is to tell the truth in order to obtain the maximum utility.

We design Step 7 as shown in the contract-based protocol in Section 3 for attendees to evaluate whether the host is trustworthy. For each meeting, the host is required to guarantee at least $M$ attendees are satisfied with the scheduled time slot either by providing them a free time slot or by offering reward $R_{F}^{\prime}$ to compensate their loss. In this way, the host's reputation is kept and attendees will continue to trust it in future meetings.

\section{Simulation Results}

We conduct empirical studies to evaluate our proposed mechanism based on a set of metrics including 1 ) privacy leakage, 2) social welfare measured by the number 
of agents free at the scheduled slot, 3) the number of rounds for convergence, 4) incentive cost which is the total rewards paid by the host.

For experiment setups, we look into a period of 5 days, 9 a.m. to 5 p.m. The whole period is split into 80 time slots of length 30 minutes. The meetings to be scheduled have different scales ranging from 6 to 12 agents. Each agent's calendar is generated with different preferences. Different cost functions are tested in the experiments and all results are averaged over 20000 cases. We compare our proposed mechanism with the following benchmarks:

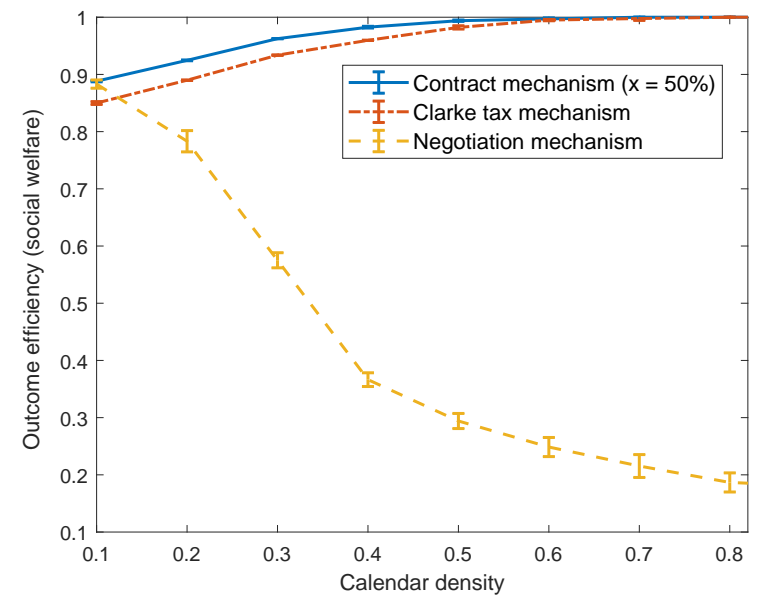

Fig. 2. Social welfare with respect to agents' average density of availability.

- Centralized meeting scheduling: a central controller is aware of all the agents' calendars and schedules the meeting in a way that the social welfare is maximized. This serves as an upper bound of the social welfare.

- Clarke-Tax based mechanism [10]: this is a typical incentive mechanism without privacy preservation. Each responder reports its availability at all time slots at the first round of negotiation. We evaluate how much privacy leakage can be saved compared to it.

- Negotiation-based distributed mechanism [7]: this line of work represents the commonly used meeting scheduling scheme without any incentive compatible guarantees. By comparing with it, we show how incentive compatibility can help to improve the performance.

We evaluate the social welfare of different mechanisms, measured by the ratio of the social welfare in the proposed scheme to the optimal social welfare in the centralized scheme, i.e., the outcome efficiency. Unlike the IC contractbased mechanism where all selfish agents are motivated to tell the truth about their preferences, the benchmarks cannot guarantee the IC property. The Clarke 
Tax mechanism can motivate all selfish responders to tell the truth about their preferences, but cannot do the same with an selfish initiator. The negotiation mechanism does not even consider IC at all.

In Fig. 2, the outcome efficiency grows with the calendar density in the contract-based and Clarke Tax mechanisms. This is because as the density increases, the number of feasible time slots becomes smaller. Thus, the initiator is more likely to select a time slot which is also the outcome of the centralized scheme, bringing a higher social welfare. For the negotiation mechanism, the outcome efficiency decreases with the increase of the calendar density. When more agents have dense calendars, it is harder for the initiator to find a feasible time slot since selfish responders keep turning down the OK time slots (as illustrated in Observation 1) which could be the output of the centralized scheme. Therefore, the social welfare decreases.

We observe that the contract mechanism serving selfish agents can achieve at least $88.31 \%$ outcome efficiency compared to the centralized scheme, which outperforms the Clarke Tax one by 3.31 percentage points, implying that the IC constraint of the initiator helps improve the social welfare. Compared to the negotiation mechanism, the outcome efficiency of the contract mechanism is significantly higher by up to 82 percentage points.

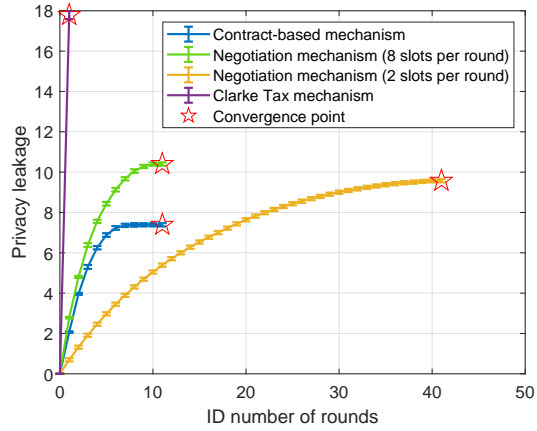

(a)

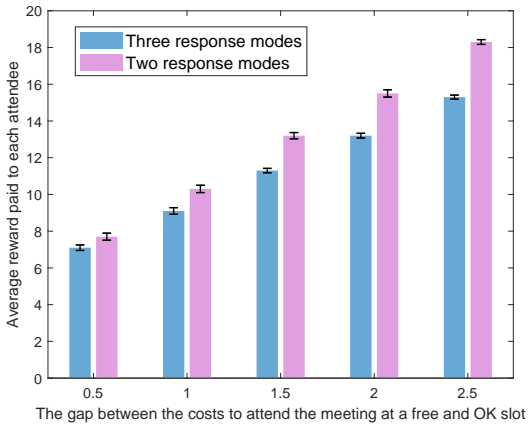

(b)

Fig. 3. a) Privacy leakage with respect to number of rounds; b) Average reward paid to each attendee with respect to the gap between cost functions $C^{F, F}$ and $C^{O, O}$

We also report on the balance between the average privacy leakage and the convergence speed as shown in Fig. 3(a). This figure presents the accumulated privacy leakage varying with the ID number of rounds starting from round 0 until convergence. Since the Clarke Tax mechanism requires all responders to report their costs of each time slot at the beginning, each agent leaks all its private information in the first round. It depicts the upper bound of privacy leakage and the lower bound of time complexity. Compared to the Clarke Tax mechanism, The contract one saves privacy leakage by $58.3 \%$. Compared with 
the negotiation mechanism, the contract-based mechanism can achieve the same level of privacy leakage with a significantly smaller number of rounds, saving $80 \%$ time costs. For the same number of rounds to converge, our mechanism efficiently reduces the privacy leakage by $26.2 \%$. This is because the contract mechanism intelligently adjusts the number of proposed time slots based on each responder's calendar density, which is different from the negotiation mechanism where only the same number of time slots are proposed to all responders regardless of their calendar densities. Benefited from this flexible propose-and-respond manner, the contract-based mechanism can achieve a better balance between privacy leakage and time complexity.

In Fig. 3(b) we evaluate the incentive cost of the contract-based mechanism, i.e., the average reward paid to each attendee, for two response modes ${ }^{5}$. In the first one, each attendee can report it is free/OK/busy. In the second mode, each attendee can only reply free or busy information. As discussed in Section 4.2, the host has to raise rewards to incentivize attendees to attend the meeting. Results in Fig. 3(b) verify our analysis and show that the average required reward in the second mode is up to $19.6 \%$ higher than that in the first mode. The gap between these cases enlarges with the gap between the cost functions $C^{F, F}-C^{O, O}$.

\section{Conclusions}

We have studied the MAMS problem where the host is required to schedule meetings via information exchange with each attendee. By optimizing the rewards and privacy leakage level, we develop a distributed incentive mechanism based on contract theory to achieve a balance between incentive compatibility, social welfare, and privacy-preservation. Through both theoretical analysis and simulation results, the following features of the proposed mechanism are verified. 1) The mechanism is guaranteed to be incentive compatible with respect to all agents. 2) Multiple types of responses are designed for each attendee such that the incentive costs are largely saved. 3) The contract mechanism can achieve a better trade-off between the privacy leakage and the convergence speed. Given the same convergence speed, the privacy leakage is reduced by $26.2 \%$ compared to a state-of-the-art negotiation mechanism; while at the same level of privacy leakage, the contract mechanism can save $80 \%$ time costs for convergence. 4) Compared to the Clarke Tax mechanism, the contract one reduces the privacy leakage by $58.3 \%$. 5) Social welfare approaches the upper bound by at least $88.31 \%$, and significantly surpasses that of the Clarke Tax mechanism the negotiation one by up to 3.31 and 82 percentage points, respectively.

Although we deliver this work in the domain of meeting scheduling, the mechanism we design contains various merits which enable other multi-agent applications that involve information exchange among agents who have privacy concerns. For future works, we will extend the constructed framework to explore its applicability to other scenarios such as e-Commerce platforms where multiple

\footnotetext{
${ }^{5}$ We do not compare with other works here since each of them has a different metric of incentive costs, which is not compatible to ours.
} 
agents trade with each other to reach a consensus while protecting their own private information.

\section{A Appendix: Proof of Proposition 1}

According to $(11 c)$, for any $(d, \hat{d})$, the following inequalities hold:

$$
\begin{aligned}
& f_{F}(d)\left(R_{F}(\hat{d})-\alpha N_{p}(\hat{d}) L(F)\right)+f_{O}(d)\left(R_{O}(\hat{d})-\alpha N_{p}(\hat{d}) L(O)\right) \leq \\
& f_{F}(d)\left(R_{F}(d)-\alpha N_{p}(d) L(F)\right)+f_{O}(d)\left(R_{O}(d)-\alpha N_{p}(d) L(O)\right), \\
& f_{F}(\hat{d})\left(R_{F}(d)-\alpha N_{p}(d) L(F)\right)+f_{O}(\hat{d})\left(R_{O}(d)-\alpha N_{p}(d) L(O)\right) \leq \\
& f_{F}(\hat{d})\left(R_{F}(\hat{d})-\alpha N_{p}(\hat{d}) L(F)\right)+f_{O}(\hat{d})\left(R_{O}(\hat{d})-\alpha N_{p}(\hat{d}) L(O)\right) .
\end{aligned}
$$

When $f_{O}(d)=f_{O}(\hat{d})$, adding $(20 a)$ and $(20 b)$ yields

$$
(\hat{d}-d)\left(R_{F}(d)-R_{F}(\hat{d})\right)+\alpha L_{F}(\hat{d}-d)\left(N_{p}(d)-N_{p}(\hat{d})\right) \leq 0 .
$$

The sufficient conditions to satisfy (21) are that both $R_{F}(d)$ and $N_{p}(d)$ are non-increasing functions. Similarly, by fixing $f_{F}(d)=f_{F}(\hat{d})$, we can learn that $R_{O}(d)$ is also a non-increasing one, which verifies $(15 a)$ and $(15 b)$.

Given $d,(20 a)$ implies that the function $y(\hat{d})=f_{F}(d)\left(R_{F}(\hat{d})-\alpha N_{p}(\hat{d}) L(F)\right)$ $+f_{O}(d)\left(R_{O}(\hat{d})-\alpha N_{p}(\hat{d}) L(O)\right)$ reaches its maximum at $\hat{d}=d$. We have

$f_{F}(d) \cdot\left[\frac{d R_{F}(d)}{d d}-\alpha L(F) \cdot \frac{d N_{p}(d)}{d d}\right]+f_{O}(d) \cdot\left[\frac{d R_{O}(d)}{d d}-\alpha L(O) \cdot \frac{d N_{p}(d)}{d d}\right]=0$.

Since it holds for all $f_{F}(d), f_{O}(d)>0$, we have $(15 c)$.

\section{B Appendix: Proof of Proposition 2}

The first and second conditions stated in Definition 1 are guaranteed by constraints (11) and (13). We now look into whether the third condition holds.

An attendee $i$ may claim to be busy at an OK time slot $t$ so as to mislead the host to schedule the meeting at time slot $t^{\prime}$ in which it is free. In this case, the desired probability that attendee $i$ wishes others to believe is $p_{0 \rightarrow i}^{d}(t)=0$. Note that slot $t^{\prime}$ is a time slot that has not been proposed yet. We consider one case that slot $t^{\prime}$ is a time slot that has not been proposed yet. The expected utility of responder $i$ when lying and telling the truth are separately given by

$$
\begin{aligned}
& U_{i}^{l i e}=\left(1-p^{l i e}\right)\left[R_{F}-C^{F, F}+\alpha\left(p_{b}-1\right)+\alpha\left(2 P_{O}-p_{b}\right)\right] \\
& U_{i}^{t r}=\left(1-p^{t r}\right)\left[R_{O}-C^{O, O}+\alpha\left(p_{b}-P_{O}\right)+\alpha\left(1-p_{b}\right)\right],
\end{aligned}
$$


where $p^{l i e}$ and $p^{t r}$ are the probability that the meeting can not be successfully scheduled in two cases, respectively. $p_{b}$ is the host's prior belief of the free probability of the attendee. In $(23 a), \alpha\left(p_{b}-1\right)$ represents the privacy leakage reporting to be free at time slot $t^{\prime}$, and $\alpha\left(2 p_{O}-p_{b}\right)$ represents the protected privacy information by lying. In $(23 b), \alpha\left(p_{b}-p_{O}\right)$ is the privacy leakage at time slot $t$, and $\theta\left(1-p_{b}\right)$ is the protected privacy information of slot $t^{\prime}$.

Since $p_{O} \leq 0.5$ and $p^{\text {lie }}>p^{t r}$, we have $E\left[U_{i}^{t r u}\right]>E\left[U_{i}^{\text {lie }}\right]$. Therefore, the attendee has no motivation to lie, which verifies the third condition.

\section{References}

1. Sen, S., Durfee, E.H.: A formal study of distributed meeting scheduling. Group Decision and Negotiation 7(3), 265-289, (1998)

2. Bolton, P., Dewatripont M.: Contract theory. MIT Press, Cambridge, MA, (2005).

3. Lee, Y.: Online Membership Incentive System \& Method. U.S. Patent Application No. $13 / 503,831$ (2012)

4. Li, Z., Yang, Z., Xie, S., Chen, W., Liu, K., Credit-based payments for fast computing resource trading in edge-assisted Internet of Things. IEEE Internet of Things Journal 6(4), 6606-6617, (2019)

5. Wang, J., Li, M., He, Y., Li, H., Xiao, K., Wang, C.: A blockchain based privacypreserving incentive mechanism in crowdsensing applications. IEEE Access 6, 1754517556, (2018)

6. Jennings, N.R., Jackson, A.J.: Agent based meeting scheduling: A design and implementation. IEEE Electronics Letters 31(5), 350-352, (1995)

7. Crawford, E, Veloso, M.: Learning to select negotiation strategies in multi-agent meeting scheduling. In: Bento C., Cardoso A., Dias G. (eds.) Progress in Artificial Intelligence. EPIA 2005. Lecture Notes in Computer Science, vol. 3808. Springer, Berlin, Heidelberg

8. Farinelli, A., Rogers, A., Petcu, A., Jennings, N. R.: Decentralised coordination of low-power embedded devices using the Max-Sum algorithm. In: Seventh International Conference on Autonomous Agents and Multi-Agent Systems (AAMAS-08), pp. 639-646

9. Hassine, A.B., Ho, T.B., Ito, T.: Meetings scheduling solver enhancement with local consistency reinforcement. In: Applied Intelligence 24(2), 143-154, (2006)

10. Tanaka, T., Farokhi, F., Langbort, C.: A faithful distributed implementation of dual decomposition and average consensus algorithms. In: 52nd IEEE Conference on Decision and Control, (2013).

11. Itoh, H.: Incentives to help in multi-agent situations. Econometrica 59(3), 611-636, (1999)

12. Di, B., Wang, T., Han, Z., Song, L.: Collaborative smartphone sensing using overlapping coalition formation games. IEEE Transactions on Mobile Computing 16(1), 30-43, (2017)

13. Xu, L., Jiang, C., Chen, Y., Ren, Y., Liu. K.J.: Privacy or utility in data collection? A contract theoretic approach. IEEE Journal of Selected Topics in Signal Processing $\mathbf{9}(7), 1256-1269,(2015)$

14. Bryon, A.E.: Applied optimal control: Optimization, estimation and control. 1st edn., Taylor \& Francis Group, NY (1975) 